\title{
An Algebraic Relation between Consimilarity and Similarity of Quaternion Matrices and Applications
}

\author{
Tongsong Jiang, ${ }^{1,2}$ Xuehan Cheng, ${ }^{3}$ and Sitao Ling ${ }^{4}$ \\ ${ }^{1}$ College of Science, Linyi University, Linyi, Shandong 276005, China \\ ${ }^{2}$ College of Mathematics and System Science, Shandong University of Science and Technology, \\ Qingdao, Shandong 266590, China \\ ${ }^{3}$ College of Mathematics and Statistics Science, Ludong University, Yantai, Shandong 264025, China \\ ${ }^{4}$ State Key Laboratory for Geomechanics and Deep Underground Engineering, Department of Mathematics, \\ China University of Mining and Technology, Xuzhou, Jiangsu 221116, China
}

Correspondence should be addressed to Sitao Ling; lingsitao2004@163.com

Received 12 May 2014; Revised 15 August 2014; Accepted 21 August 2014; Published 1 September 2014

Academic Editor: Fan Min

Copyright (C) 2014 Tongsong Jiang et al. This is an open access article distributed under the Creative Commons Attribution License, which permits unrestricted use, distribution, and reproduction in any medium, provided the original work is properly cited.

\begin{abstract}
This paper, by means of complex representation of a quaternion matrix, discusses the consimilarity of quaternion matrices, and obtains a relation between consimilarity and similarity of quaternion matrices. It sets up an algebraic bridge between consimilarity and similarity, and turns the theory of consimilarity of quaternion matrices into that of ordinary similarity of complex matrices. This paper also gives algebraic methods for finding coneigenvalues and coneigenvectors of quaternion matrices by means of complex representation of a quaternion matrix.
\end{abstract}

\section{Introduction}

An antilinear operator $T$ is a mapping from one complex vector space $V$ into another $W$, which is additive and conjugate homogeneous, that is, for all $\alpha, \beta \in V$ and any complex number $a, T(\alpha+\beta)=T(\alpha)+T(\beta), T(a \alpha)=\bar{a} T(\alpha)$ in which $\bar{a}$ is the conjugate of $a$. Two $n \times n$ complex matrices $A, B$ are said to be consimilar if $S^{-1} A \bar{S}=B$ for some $n \times n$ nonsingular complex matrix $S$. Consimilarity of complex matrices arises as a result of studying an antilinear operator referred to different bases in complex vector spaces, and the theory of consimilarity of complex matrices plays an important role in quantum mechanics $[1,2]$. Complex consimilarity is an equivalent relation and has been studied [2-5].

In recent years, applications of quaternion matrices are getting more and more important and extensive in quantum mechanics, rigid mechanics, and control theory [6-17]; it is becoming more and more necessary to study the theory and methods of quaternion matrices. In paper [18], the author introduced concepts of consimilarity of quaternion matrices.
If $A$ and $B$ are both quaternion matrices of $n \times n$, they are said to be consimilar if $S^{-1} A \widetilde{S}=B$ holds for some $n \times n$ nonsingular quaternion matrix $S$. Write $A \stackrel{\mathcal{s}}{\sim} B$ if $A$ is similar to $B, A \stackrel{\mathcal{c S}}{\sim} B$ if $A$ is consimilar to $B$, and $A \stackrel{p s}{\sim} B$ if $A$ is permutation similar to $B$. Permutation similarity is both a similarity and consimilarity relations. A quaternion $\lambda$ is said to be the right coneigenvalue attributed to $A$ if the con-eigenequation $A \widetilde{\alpha}=$ $\alpha \lambda$ holds. The consimilarity of quaternion matrices is natural extension of that of complex matrices and will have potential applications in the study of theory and numerical computations in modern quantum mechanics, and so forth.

Let $\mathbf{R}$ be the real number field, $\mathbf{C}=\mathbf{R} \oplus \mathbf{R} i$ the complex number field, and $\mathbf{H}=\mathbf{R} \oplus \mathbf{R} i \oplus \mathbf{R} j \oplus \mathbf{R} k$ the quaternion field, where $i j=-j i=k, i^{2}=j^{2}=k^{2}=-1 . \mathbf{F}^{m \times n}$ denotes the set of all $m \times n$ matrices on a field $\mathbf{F}$. For $A \in \mathbf{H}^{m \times n}$, let $A^{T}$ be the transpose of $A$ and $\bar{A}$ the conjugate matrix of $A$. For a quaternion $\alpha=a_{1}+a_{2} i+a_{3} j+a_{4} k$ with $a_{t} \in \mathbf{R}$, we use $\bar{\alpha}=a_{1}-a_{2} i-a_{3} j-a_{4} k$ and $\widetilde{\alpha}=a_{1}-a_{2} i+a_{3} j-a_{4} k$ to denote the conjugate and $j$-conjugate of $\alpha$, respectively. 
Let $A=A_{1}+A_{2} i+A_{3} j+A_{4} k$, where $A_{s} \in \mathbf{R}^{m \times n}$; define $\widetilde{A}=-j A j=A_{1}-A_{2} i+A_{3} j-A_{4} k$ to be $j$-conjugate of $A$. It is easy to verify that $j A j=-\widetilde{A}, \widetilde{\widetilde{A}}=A$, and

$$
\widetilde{A+B}=\widetilde{A}+\widetilde{B}, \quad \widetilde{A C}=\widetilde{A C},
$$

for any two $n \times n$ matrices $A, B$ and a $n \times n$ matrix $C$ with quaternion entries. Obviously, $\widetilde{A}=\bar{A}$ if $A$ is a complex matrix.

By means of real representation of complex matrices in paper [5], we studied the properties of consimilarity of complex matrices and gave a relation between consimilarity and similarity of complex matrices. This paper, by means of a complex representation of a quaternion matrix, studies the relation between consimilarity and similarity of quaternion matrices and derives an algebraic relation between consimilarity and similarity on quaternion field. Finally this paper gives an application on coneigenvalues and coneigenvectors of quaternion matrices.

\section{Preliminaries}

For a quaternion matrix $A$ of dimension $n \times m$, denote by $A=B_{1}+B_{2} i+B_{3} j+B_{4} k \equiv A_{1}+A_{2} j$, where $A_{1}=B_{1}+B_{2} i$, $A_{2}=B_{3}+B_{4} i$. The complex representation of the quaternion matrix $A$ is defined [19] to be

$$
A^{\sigma}=\left[\begin{array}{cc}
A_{2} & -A_{1} \\
\bar{A}_{1} & \bar{A}_{2}
\end{array}\right] \in \mathbf{C}^{2 n \times 2 m} ;
$$

the complex matrix $A^{\sigma}$ is known as complex representation of the quaternion matrix $A$. It is easy to verify that $\overline{A^{\sigma}}=(\widetilde{A})^{\sigma}$.

Let $A, B \in \mathbf{H}^{n \times m}, C \in \mathbf{H}^{m \times p}, a \in \mathbf{R}$. Then by the definition of complex representation we easily get the following results:

$$
\begin{gathered}
(A+B)^{\sigma}=A^{\sigma}+B^{\sigma}, \quad(a A)^{\sigma}=a A^{\sigma} ; \\
Q_{n}^{T} A^{\sigma} Q_{m}=\overline{A^{\sigma}}=(\widetilde{A})^{\sigma} ; \\
(A C)^{\sigma}=A^{\sigma} Q_{m} C^{\sigma}=A^{\sigma}(\widetilde{C})^{\sigma} Q_{p}=Q_{n}(\widetilde{A})^{\sigma} C^{\sigma},
\end{gathered}
$$

in which $Q_{t}=\left[\begin{array}{cc}0 & I_{t} \\ -I_{t} & 0\end{array}\right]$ with $I_{t}$ being the identity matrix, and $Q_{t}^{2}=-I_{2 t}, Q_{t} Q_{t}^{T}=Q_{t}^{T} Q_{t}=I_{2 t}$. Clearly, from (5) we know that a quaternion matrix $A$ is nonsingular if and only if complex representation matrix $A^{\sigma}$ is nonsingular.

For $A \in \mathbf{H}^{n \times n}$ and $\alpha \in \mathbf{C}^{2 n \times 1}$, if $A^{\sigma} \alpha=\lambda \alpha$, then by (4) we have

$$
\bar{A}^{\sigma}\left(Q_{n}^{T} \alpha\right)=\lambda\left(Q_{n}^{T} \alpha\right) \Longleftrightarrow A^{\sigma}\left(Q_{n}^{T} \bar{\alpha}\right)=\bar{\lambda}\left(Q_{n}^{T} \bar{\alpha}\right) .
$$

This means that the eigenvalues of complex representation $A^{\sigma}$ appear in conjugate pairs.

In the same manner, for a Jordan block $J_{r}(\lambda)$ of an eigenvalue $\lambda$, if $A^{\sigma} X=X J_{r}(\lambda)$, then by (4) we have

$$
\bar{A}^{\sigma}\left(Q_{n}^{T} X\right)=\left(Q_{n}^{T} X\right) J_{r}(\lambda) \Longleftrightarrow A^{\sigma}\left(Q_{n}^{T} \bar{X}\right)=\left(Q_{n}^{T} \bar{X}\right) J_{r}(\bar{\lambda}) .
$$

And this means Jordan blocks $J_{r}(\lambda)$ of complex representation $A^{\sigma}$ appear in conjugate pairs.

From the statement above we have the following result.
Proposition 1. Let $A \in \mathbf{H}^{n \times n}$. Then

(1) the real eigenvalues of complex representation $A^{\sigma}$ appear in pairs, and the imaginary eigenvalues of complex representation $A^{\sigma}$ appear in conjugate pairs; that is, if complex eigenvalue $\lambda$ is an eigenvalue of $A^{\sigma}$, then complex eigenvalue $\bar{\lambda}$ is also an eigenvalue of $A^{\sigma}$,

(2) the real Jordan blocks $J_{r}(\lambda)$ of complex representation $A^{\sigma}$ appear in pairs, and the imaginary Jordan blocks $J_{r}(\lambda)$ of complex representation $A^{\sigma}$ appear in conjugate pairs; that is, if $J_{r}(\lambda)$ is a Jordan block of complex representation $A^{\sigma}$ related to complex eigenvalue $\lambda$, then $J_{r}(\bar{\lambda})$ is a Jordan block of complex representation $A^{\sigma}$ related to complex eigenvalue $\bar{\lambda}$.

The following Proposition 2 comes from the fact that $j A j=-\widetilde{A},\left(J_{r}(a+b i)\right) \overline{j(i+j)}=(i+j) J_{r}(-b+a j)$ and $\left(\left(A_{1}+\right.\right.$ $\left.\left.A_{2} i\right) j\right) \widetilde{(i+j)}=(i+j)\left(-A_{2}+A_{1} j\right)$ by direct calculation for real numbers $a$ and $b$, and real matrices $A_{1}$ and $A_{2}$.

Proposition 2. Let $A$ be a quaternion matrix. Then

(1) $A j=j \widetilde{A}$; this means that $A$ is consimilar to $\widetilde{A}$; that is, $A \stackrel{c s}{\sim} \widetilde{A}$

(2) if $J=J_{r}(a+b i)$ is a Jordan block, $a, b \in \mathbf{R}$, then $J j \stackrel{c s}{\sim}$ $J_{r}(-b+a j)$

(3) if $A=A_{1}+A_{2} i$ is a complex matrix, $A_{1}, A_{2} \in \mathbf{R}^{n \times n}$, then

$$
A j \stackrel{c s}{\sim}-A_{2}+A_{1} j, \quad A j \stackrel{c s}{\sim}\left(A_{1}+A_{2} j\right) j .
$$

Based on the quaternion field $\mathbf{H}$, the author in [18] gave the following results.

Proposition 3 (see [18] ). If $A, B \in \mathbf{H}^{n \times n}$, then

$$
A \stackrel{\mathcal{c s}}{\sim} B \Longleftrightarrow j A \stackrel{s}{\sim} j B \Longleftrightarrow A j \stackrel{s}{\sim} B j \Longleftrightarrow j A \stackrel{s}{\sim} B j .
$$

Proposition 4 (see [18] ). If $A \in \mathbf{H}^{n \times n}$, then

$$
A \stackrel{c s}{\sim} J_{n_{1}}\left(\lambda_{1}\right) \oplus J_{n_{2}}\left(\lambda_{2}\right) \oplus \cdots \oplus J_{n_{r}}\left(\lambda_{r}\right) \equiv J_{A}^{c},
$$

in which $\lambda_{t}=a_{t}+b_{t} j$ are right coneigenvalues of $A, a_{t}, b_{t}$ are real numbers, and $a_{t} \geq 0$. $J_{A}^{c}$ is uniquely determined by $A$ up to the order of Jordan blocks $J_{n_{t}}\left(\lambda_{t}\right)$, and $J_{A}^{c}$ is said to be the Jordan canonical form of $A$ under consimilarity.

\section{An Algebraic Relation between Consimilarity and Similarity}

This section gives an algebraic relation between consimilarity and similarity of quaternion matrices by means of the complex representation in (2).

Let $A, B \in \mathbf{H}^{n \times n}$ be two quaternion matrices. If $A$ is consimilar to $B$, then there exists a nonsingular quaternion matrix $S$ such that $A S=\widetilde{S} B$, by (5) $A^{\sigma} Q_{n} S^{\sigma}=Q_{n} S^{\sigma} B^{\sigma}$. 
This means that if $A$ is consimilar to $B$, then $A^{\sigma}$ is similar to $B^{\sigma}$.

Conversely, if $A^{\sigma}$ is similar to $B^{\sigma}$, then $A^{\sigma}$ and $B^{\sigma}$ have the same eigenvalues. By Proposition 1 , let $\lambda_{1}, \bar{\lambda}_{1}, \ldots, \lambda_{r}, \bar{\lambda}_{r}$ be all eigenvalues and $J_{n_{1}}\left(\lambda_{1}\right), J_{n_{1}}\left(\bar{\lambda}_{1}\right), \ldots, J_{n_{r}}\left(\lambda_{r}\right), J_{n_{r}}\left(\bar{\lambda}_{r}\right)$ corresponding Jordan blocks. There exists a complex and full-rank matrix $X_{j}$ by [20, chapter 6.7] such that $A^{\sigma} X_{t}=X_{t} J_{n_{t}}\left(\lambda_{t}\right)$, $t=1, \ldots, r$. Then by (4), we have

$$
A^{\sigma} X_{t}=X_{t} J_{n_{t}}\left(\lambda_{t}\right), \quad A^{\sigma}\left(Q_{n}^{T} \bar{X}_{t}\right)=\left(Q_{n}^{T} \bar{X}_{t}\right) J_{n_{t}}\left(\bar{\lambda}_{t}\right),
$$

and (11) is equivalent to

$$
A^{\sigma}\left(X_{t}, Q_{n}^{T} \bar{X}_{t}\right)=\left(X_{t}, Q_{n}^{T} \bar{X}_{t}\right)\left[\begin{array}{cc}
J_{n_{t}}\left(\lambda_{t}\right) & 0 \\
0 & J_{n_{t}}\left(\bar{\lambda}_{t}\right)
\end{array}\right]
$$

Therefore there exists a nonsingular complex matrix $T=$ $\left(Z, Q_{n}^{T} \bar{Z}\right) \in \mathbf{C}^{2 n \times 2 n}, Z \in \mathbf{C}^{2 n \times n}$ such that

$$
T^{-1} A^{\sigma} T=(J j)^{\sigma}=\left[\begin{array}{cc}
\sum_{t} \oplus J_{n_{t}}\left(\lambda_{t}\right) & 0 \\
0 & \sum_{t} \oplus J_{n_{t}}\left(\bar{\lambda}_{t}\right)
\end{array}\right],
$$

in which $J=\sum_{t} \oplus J_{n_{t}}\left(\lambda_{t}\right), \lambda_{t}=a_{t}+b_{t} i, a_{t}, b_{t}$ are real numbers, and $b_{t} \geq 0$.

Let $Z=\left[\begin{array}{l}Z_{1} \\ Z_{2}\end{array}\right], Z_{1}, Z_{2} \in \mathbf{C}^{n \times n}$, and $S=\bar{Z}_{2}+Z_{1} j$. Then $T=\left[\begin{array}{cc}Z_{1} & -\bar{Z}_{2} \\ Z_{2} & \bar{Z}_{1}\end{array}\right]=S^{\sigma}$. From the nonsingular complex matrix $T$, we get $S$ is a nonsingular quaternion matrix; by (1) and (5), (13) is equivalent to

$$
\begin{aligned}
A^{\sigma} S^{\sigma}=S^{\sigma}(J j)^{\sigma} & \Longleftrightarrow(A \widetilde{S})^{\sigma}=(S \widetilde{(J j)})^{\sigma} \\
& \Longleftrightarrow A \widetilde{S}=S \widetilde{J} j=S \bar{J} j .
\end{aligned}
$$

This means that $A \stackrel{c s}{\sim} \bar{J} j$. Let $\lambda_{t}=a_{t}+b_{t} i, a_{t}, b_{t}$ are real numbers, and $b_{t} \geq 0$, and $\bar{J}=\sum_{t} \oplus J_{n_{t}}\left(\bar{\lambda}_{t}\right)=\sum_{t} \oplus J_{n_{t}}\left(a_{t}-b_{t} i\right)$. Then by Proposition 2 we have $\bar{J} j \stackrel{c s}{\sim} \sum_{t} \oplus J_{n_{t}}\left(b_{t}+a_{t} j\right)$, and we have $A \stackrel{\text { cs }}{\sim} \sum_{t} \oplus J_{n_{t}}\left(b_{t}+a_{t} j\right)$. Similarly $B \stackrel{c s}{\sim} \sum_{t} \oplus J_{n_{t}}\left(b_{t}+a_{t} j\right)$. Therefore $A \stackrel{c s}{\sim} B$.

The statement above implies the following result.

Theorem 5. Let $A, B \in \mathbf{H}^{n \times n}$. Then $A$ is consimilar to $B$ if and only if complex representation matrix $A^{\sigma}$ is similar to complex representation matrix $B^{\sigma}$; that is, $A \stackrel{\text { cs }}{\sim} B$ if and only if $A^{\sigma} \stackrel{\stackrel{s}{\sim}}{\sim} B^{\sigma}$.

Combining Proposition 3 and Theorem 5, we get the following result.

Corollary 6. Let $A, B \in \mathbf{H}^{n \times n}$. Then

$$
\begin{aligned}
A \stackrel{c s}{\sim} B & \Longleftrightarrow A j \stackrel{s}{\sim} B j \\
& \Longleftrightarrow j A \stackrel{s}{\sim} j B \Longleftrightarrow j A \stackrel{s}{\sim} B j \Longleftrightarrow A^{\sigma} \sim B^{\sigma} .
\end{aligned}
$$

The proof of the Theorem 5 is constructive, and the following results come from the proof above.

Corollary 7. Let $A \in \mathbf{H}^{n \times n}$. Then the following statements are equivalent.

(1) The Jordan canonical form of complex matrix $A^{\sigma}$ under similarity is

$$
J=\sum_{t} \oplus J_{n_{t}}\left(\lambda_{t}\right) \oplus \sum_{t} \oplus J_{n_{t}}\left(\bar{\lambda}_{t}\right)
$$

where $\lambda_{t}=a_{t}+b_{t} i, a_{t}, b_{t} \in \mathbf{R}, b_{t} \geq 0, t=1, \ldots, r$, are eigenvalues of $A^{\sigma}$.

(2) The Jordan canonical form of quaternion matrix $A$ under consimilarity is

$$
J^{c}=\sum_{t} \oplus J_{n_{t}}\left(\mu_{t}\right)
$$

where $\mu_{t}=b_{t}+a_{t} j, a_{t}, b_{t} \in \mathbf{R}, b_{t} \geq 0, t=1, \ldots, r$, are coneigenvalues of $A$.

Corollary 8. Let $A \in \mathbf{H}^{n \times n}$. Then

$$
A \stackrel{c s}{\sim} J_{n_{1}}\left(\mu_{1}\right) \oplus J_{n_{2}}\left(\mu_{2}\right) \cdots \oplus J_{n_{r}}\left(\mu_{r}\right) \equiv J^{c}
$$

where $\mu_{t}=b_{t}+a_{t} j=(1 / 2 i)\left(\lambda_{t}-\bar{\lambda}_{t}\right)+(1 / 2)\left(\lambda_{t}+\bar{\lambda}_{t}\right) j$ are coneigenvalues of $A, \lambda_{t}=a_{t}+b_{t} i, a_{t}, b_{t} \in \mathbf{R}, b_{t} \geq 0, t=1, \ldots, r$, in which $\lambda_{1}, \bar{\lambda}_{1}, \ldots, \lambda_{r}, \bar{\lambda}_{r}$ are all eigenvalues of complex representation $A^{\sigma}$, and $J_{n_{1}}\left(\lambda_{1}\right), J_{n_{1}}\left(\bar{\lambda}_{1}\right), \ldots, J_{n_{r}}\left(\lambda_{r}\right), J_{n_{r}}\left(\bar{\lambda}_{r}\right)$ corresponding Jordan blocks in (16). $J^{c}$ is uniquely determined by $A$ up to the order of Jordan blocks $J_{n_{t}}\left(\mu_{t}\right)$, and $J^{c}$ is said to be the Jordan canonical form of A under consimilarity.

Corollary 9. Let $A \in \mathbf{H}^{n \times n}$. Then $A$ has at least a coneigenvalue $\mu_{t}=\operatorname{Re}\left(\mu_{t}\right)+\operatorname{Im}\left(\mu_{t}\right) j, \operatorname{Re}\left(\mu_{t}\right) \geq 0$. If $\lambda_{1}, \bar{\lambda}_{1}, \ldots, \lambda_{r}, \bar{\lambda}_{r}$, $\lambda_{t}=a_{t}+b_{t} i, a_{t}, b_{t} \in \mathbf{R}, b_{t} \geq 0, t=1, \ldots, r$, are all eigenvalues of complex representation $A^{\sigma}$, then $\mu_{1}, \ldots, \mu_{r}$ are coneigenvalues of quaternion matrix $A$, in which

$$
\mu_{t}=b_{t}+a_{t} j=\frac{1}{2 i}\left(\lambda_{t}-\bar{\lambda}_{t}\right)+\frac{1}{2}\left(\lambda_{t}+\bar{\lambda}_{t}\right) j
$$

and for any nonzero quaternion $p, \widetilde{p}^{-1} \mu_{t} p, t=1, \ldots, r$, are all coneigenvalues of quaternion matrix $A$.

The following result comes immediately from Theorem 5 and the definition of complex representation in (2).

Corollary 10. Let $A, B \in \mathbf{C}^{n \times n}$. Then $A$ is consimilar to $B$ if and only if $\left[\begin{array}{cc}0 & -A \\ A & 0\end{array}\right]$ is similar to $\left[\begin{array}{cc}0 & -B \\ B & 0\end{array}\right]$.

\section{Algebraic Methods and Applications}

This section gives an algebraic method for relation between consimilarity and similarity of quaternion matrices by means of complex representation and obtains an algebraic method for coneigenvalues and coneigenvectors for quaternion matrices as an application. 
Let $A \in \mathbf{H}^{n \times n}, B \in \mathbf{H}^{m \times m}$. Suppose there exists a complex matrix $T \in \mathbf{C}^{2 n \times 2 m}$ such that $A^{\sigma} T=T B^{\sigma}$. By (4)

$$
A^{\sigma} T=T B^{\sigma} \Longleftrightarrow A^{\sigma}\left(Q_{n} \bar{T} Q_{m}^{T}\right)=\left(Q_{n} \bar{T} Q_{m}^{T}\right) B^{\sigma} .
$$

Let $\widehat{T}=(1 / 2)\left(T+Q_{n} \bar{T} Q_{m}^{T}\right)$. Then

$$
A^{\sigma} \widehat{T}=\widehat{T} B^{\sigma} .
$$

Let

$$
T=\left[\begin{array}{ll}
T_{11} & T_{12} \\
T_{21} & T_{22}
\end{array}\right]
$$

where $T_{k l} \in \mathbf{C}^{n \times m}$. It is easy to get by direct calculation

$$
\widehat{T}=\left[\begin{array}{cc}
\widehat{T}_{1} & -\widehat{T}_{2} \\
\bar{T}_{2} & \bar{T}_{1}
\end{array}\right],
$$

where

$$
\widehat{T}_{1}=\frac{1}{2}\left(T_{11}+\bar{T}_{22}\right), \quad \widehat{T}_{2}=\frac{1}{2}\left(T_{21}-\bar{T}_{12}\right) .
$$

From (23) we construct a complex matrix $S$ using the identities $j \widehat{T}_{t}=\widehat{T}_{t} j$ and $j \widehat{T}_{t} j=-\widehat{T}_{t}$

$$
\begin{aligned}
S & =\widehat{T}_{2}+\widehat{T}_{1} j=\frac{1}{2}\left(I_{n}, j I_{n}\right) \widehat{T}\left[\begin{array}{c}
I_{m} \\
-j I_{m}
\end{array}\right] \\
& =\frac{1}{4}\left(I_{n}, j I_{n}\right)\left(T+Q_{n} \bar{T} Q_{m}^{T}\right)\left[\begin{array}{c}
I_{m} \\
-j I_{m}
\end{array}\right] .
\end{aligned}
$$

Clearly $S^{\sigma}=\widehat{T}$. Therefore by $(5)$ and $j B j=-\widetilde{B},(21)$ is equivalent to

$$
\begin{aligned}
A^{\sigma} S^{\sigma}=S^{\sigma} B^{\sigma} & \Longleftrightarrow(A \widetilde{S})^{\sigma}=(S \widetilde{B})^{\sigma} \\
& \Longleftrightarrow A \widetilde{S}=S \widetilde{B} \Longleftrightarrow A \widetilde{S j}=S j B .
\end{aligned}
$$

The statement above implies the following result.

Proposition 11. Let $A \in \mathbf{H}^{n \times n}, B \in \mathbf{H}^{m \times m}$. Then there exists a quaternion matrix $S \in \mathbf{H}^{n \times m}$ such that $A \widetilde{S}=S B$ if and only if there exists a complex matrix $T \in \mathbf{H}^{2 n \times 2 m}$ such that $A^{\sigma} T=$ $T B^{\sigma}$. In which case, there exists a complex matrix $T$ such that $A^{\sigma} T=T B^{\sigma}$; let

$$
S=\frac{1}{4}\left(I_{n}, j I_{n}\right)\left(T+Q_{n} \bar{T} Q_{m}^{T}\right)\left[\begin{array}{c}
I_{m} \\
-j I_{m}
\end{array}\right] j .
$$

Then $S$ is a quaternion matrix and $A \widetilde{S}=S B$.

By Theorem 5 and the statement above we have the following result.

Corollary 12. Let $A, B \in \mathbf{H}^{n \times n}$. Then $A$ is consimilar to $B$ if and only if $A^{\sigma}$ is similar to $B^{\sigma}$; that is, $A \stackrel{\text { cs }}{\sim} B$ if and only if
$A^{\sigma} \stackrel{s}{\sim} B^{\sigma}$. In which case, there exists a nonsingular complex matrix $T$ such that $A^{\sigma} T=T B^{\sigma}$; let

$$
S=\frac{1}{4}\left(I_{n}, j I_{n}\right)\left(T+Q_{n} \bar{T} Q_{n}^{T}\right)\left[\begin{array}{c}
I_{n} \\
-j I_{n}
\end{array}\right] j .
$$

Then $S$ is a quaternion matrix and $A \widetilde{S}=S B$, and if $S$ is a nonsingular, then $A \stackrel{\text { cs }}{\sim} B$.

Corollary 12 gives an algebraic method for the relation between consimilarity and similarity of quaternion matrices by means of complex representation and turns theory of consimilarity of quaternion matrices into that of ordinary similarity of complex representation matrices.

As a special case of Corollary 12, we give an algebraic method for relation between coneigenvalues and coneigenvectors of a quaternion matrix and corresponding eigenvalues and eigenvectors of the complex representation matrix as follows.

Corollary 13. Let $A \in \mathbf{H}^{n \times n}, \lambda \in \mathbf{H}$. Then there exists a vector $\alpha \in \mathbf{H}^{n \times 1}$ such that $A \widetilde{\alpha}=\alpha \lambda$ if and only if there exists a vector $\beta \in \mathbf{H}^{2 n \times 1}$ such that $A^{\sigma} \beta=\beta \lambda^{\sigma}$. In which case, there exists a vector $\beta \in \mathbf{H}^{2 n \times 1}$ such that $A^{\sigma} \beta=\beta \lambda^{\sigma}$; let

$$
\alpha=\frac{1}{4}\left(I_{n}, j I_{n}\right)\left(\beta+Q_{n} \bar{\beta} Q_{1}^{T}\right)\left[\begin{array}{c}
1 \\
-j
\end{array}\right] j .
$$

Then $A \tilde{\alpha}=\alpha \lambda$.

Now we give algebraic methods for finding coneigenvalues and coneigenvectors of a quaternion matrix by means of complex representation.

For $A \in \mathbf{H}^{n \times n}$, if $\mu$ is a complex eigenvalue of $A^{\sigma}$ with $A^{\sigma} \beta=\beta \mu, \beta \in \mathbf{C}^{2 n \times 1}, \beta \neq 0$, then by (11) and (12), $A^{\sigma} \beta=\beta \mu$, $A^{\sigma}\left(Q_{n}^{T} \bar{\beta}\right)=\left(Q_{n}^{T} \bar{\beta}\right) \bar{\mu}$, and

$$
A^{\sigma}\left(\beta, Q_{n}^{T} \bar{\beta}\right)=\left(\beta, Q_{n}^{T} \bar{\beta}\right)\left[\begin{array}{cc}
\mu & 0 \\
0 & \bar{\mu}
\end{array}\right] \Longleftrightarrow A^{\sigma} T=T(\mu j)^{\sigma},
$$

in which $T=\left(\beta, Q_{n}^{T} \bar{\beta}\right) \in \mathbf{C}^{2 n \times 2}$. By Corollary 13 , let

$$
\alpha=\frac{1}{4}\left(I_{n}, j I_{n}\right)\left(\beta+Q_{n} \bar{\beta} Q_{1}^{T}\right)\left[\begin{array}{c}
1 \\
-j
\end{array}\right] j .
$$

Then $A \tilde{\alpha}=\alpha(\bar{\mu} j)$. This means that $\bar{\mu} j$ is a coneigenvalue of $A$ and if $\alpha \neq 0$, then $\alpha$ is a coneigenvector related to coneigenvalue $\bar{\mu} j$.

By the statement above we get the following result.

Corollary 14. Let $A \in \mathbf{H}^{n \times n}$. If $\mu$ is a complex eigenvalue of complex representation $A^{\sigma}$ and $\beta$ is eigenvector related to $\mu$ with $A^{\sigma} \beta=\beta \mu$, let

$$
\alpha=\frac{1}{4}\left(I_{n}, j I_{n}\right)\left(\beta+Q_{n} \bar{\beta} Q_{1}^{T}\right)\left[\begin{array}{c}
1 \\
-j
\end{array}\right] j .
$$

Then $A \tilde{\alpha}=\alpha(\bar{\mu} j)$. This means if $\alpha \neq 0$, then $\bar{\mu} j$ is a coneigenvalue of $A$ and $\alpha$ is a coneigenvector related to coneigenvalue $\bar{\mu} j$. 
Moreover, let $\mu=a+b i, a, b \in \mathbf{R}$, and $b \geq 0$. Then by Proposition 2 and the fact that $((a+b i) j) \widetilde{(i+j)}=(i+j)(-b+$ aj) we have

$$
\begin{aligned}
A \widetilde{\beta} & =\beta(\bar{\mu} j) \Longleftrightarrow A(\widetilde{\beta(i+j)}) \\
& =(\beta(i+j))(b+a j) \Longleftrightarrow A \widetilde{\gamma}=\gamma \lambda,
\end{aligned}
$$

in which $\lambda=b+a j$ and $\gamma=\beta(i+j)$. That is $\lambda$ is a coneigenvalue of quaternion matrix $A$, and $\gamma$ is a coneigenvector related to $\lambda$.

By Corollary 14 and the statement above we have the following result.

Theorem 15. Let $A \in \mathbf{H}^{n \times n}$. If $\mu$ is a complex eigenvalue of complex representation $A^{\sigma}$ and $\beta$ is an eigenvector related to $\mu$ with $A^{\sigma} \beta=\beta \mu$, let

$$
\begin{gathered}
\lambda=\frac{1}{2 i}(\mu-\bar{\mu})+\frac{1}{2}(\mu+\bar{\mu}) j, \\
\alpha=\frac{1}{4}\left(I_{n}, j I_{n}\right)\left(\beta+Q_{n} \bar{\beta} Q_{1}^{T}\right)\left[\begin{array}{c}
1 \\
-j
\end{array}\right] j, \\
\gamma=\alpha(i+j)=\frac{1}{4}\left(I_{n}, j I_{n}\right)\left(\beta+Q_{n} \bar{\beta} Q_{1}^{T}\right)\left[\begin{array}{c}
1 \\
-j
\end{array}\right] j(i+j) .
\end{gathered}
$$

Then $A \widetilde{\alpha}=\alpha(\bar{\mu} j)$ and $A \tilde{\gamma}=\gamma \lambda$. These mean that $(\bar{\mu} j)$ and $\lambda$ are two coneigenvalues of quaternion matrix $A$, and if $\beta \neq 0$, then $\alpha$ and $\gamma$ are corresponding coneigenvectors related to $(\bar{\mu} j)$ and $\lambda$, respectively. Moreover $\tilde{p}^{-1} \lambda p$ are right coneigenvalues of $A$, and $\gamma \widetilde{p}$ is corresponding coneigenvector for any nonzero quaternion $p$.

\section{Conflict of Interests}

The authors declare that there is no conflict of interests regarding the publication of this paper.

\section{Acknowledgments}

The authors would like to express their sincere thanks to the referees for the careful reading and very helpful comments on the earlier versions of this paper. This paper is supported by the National Natural Science Foundation of China (11301529 and 11301252) and Postdoctoral Science Foundation of China (2013M540472).

\section{References}

[1] J. J. Sakurai, Modern Quantum Mechanics, Benjamin Cummings, Menlo Park, Calif, USA, 1985.

[2] R. A. Horn and C. R. Johnson, Matrix Analysis, Cambridge University Press, New York, NY, USA, 1990.

[3] Y. P. Hong, Consimilarity: theory and applications [doctoral dissertation], Johns Hopkins University, 1985.

[4] Y. P. Hong and R. A. Horn, "A canonical form for matrices under consimilarity," Linear Algebra and Its Applications, vol. 102, pp. 143-168, 1988.
[5] T. Jiang, X. Cheng, and L. Chen, "An algebraic relation between consimilarity and similarity of complex matrices and its applications," Journal of Physics A, vol. 39, no. 29, pp. 9215-9222, 2006.

[6] S. L. Adler, "Quaternionic quantum field theory," Physical Review Letters, vol. 55, no. 8, pp. 783-786, 1985.

[7] S. L. Adler, "Quaternionic quantum field theory," Communications in Mathematical Physics, vol. 104, no. 4, pp. 611-656, 1986.

[8] S. L. Adler, "Scattering and decay theory for quaternionic quantum mechanics, and the structure of induced $T$ nonconservation," Physical Review D Particles and Fields, vol. 37, no. 12, pp. 3654-3662, 1988.

[9] S. L. Adler, Quaternionic Quantum Mechanics and Quantum Fields, Oxford University Press, New York, NY, USA, 1994.

[10] A. J. Davies and B. H. J. McKellar, "Nonrelativistic quaternionic quantum mechanics in one dimension," Physical Review A, vol. 40, no. 8, pp. 4209-4214, 1989.

[11] A. J. Davies and B. H. J. McKellar, "Observability of quaternionic quantum mechanics," Physical Review A, vol. 46, no. 7, pp. 36713675, 1992.

[12] A. G. Klein, "Schrödinger inviolate: neutron optical searches for violations of quantum mechanics," Physica B, vol. 151, no. 1-2, pp. 44-49, 1988.

[13] A. Peres, "Proposed test for complex versus quaternion quantum theory," Physical Review Letters, vol. 42, no. 11, pp. 683-686, 1979.

[14] P. Sutcliffe, "Instantons and the buckyball," High Energy Phys. Theory, vol. 16, p. 157, 2003.

[15] S. de Leo and P. Rotelli, "Odd-dimensional translation between complex and quaternionic quantum mechanics," Progress of Theoretical Physics, vol. 96, no. 1, pp. 247-255, 1996.

[16] S. de Leo and G. Scolarici, "Right eigenvalue equation in quaternionic quantum mechanics," Journal of Physics A, vol. 33, no. 15, pp. 2971-2995, 2000.

[17] S. de Leo, G. Scolarici, and L. Solombrino, "Quaternionic eigenvalue problem," Journal of Mathematical Physics, vol. 43, no. 11, pp. 5815-5829, 2002.

[18] H. Liping, "Consimilarity of quaternion matrices and complex matrices," Linear Algebra and Its Applications, vol. 331, no. 1-3, pp. 21-30, 2001.

[19] T. Jiang and S. Ling, "Algebraic methods for condiagonalization under consimilarity of quaternion matrices in quaternionic quantum mechanics," Advances in Applied Clifford Algebras, vol. 23, no. 2, pp. 405-415, 2013.

[20] P. Lancaster and M. Tismenetsky, The Theory of Matrices with Applications, Academic Press, New York, NY, USA, 2nd edition, 1985. 


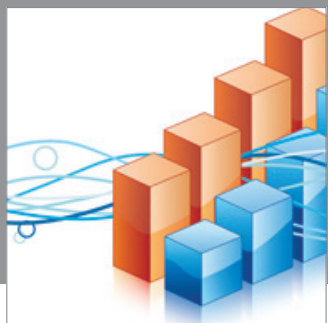

Advances in

Operations Research

mansans

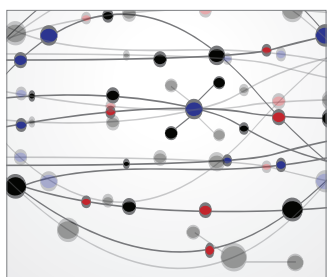

The Scientific World Journal
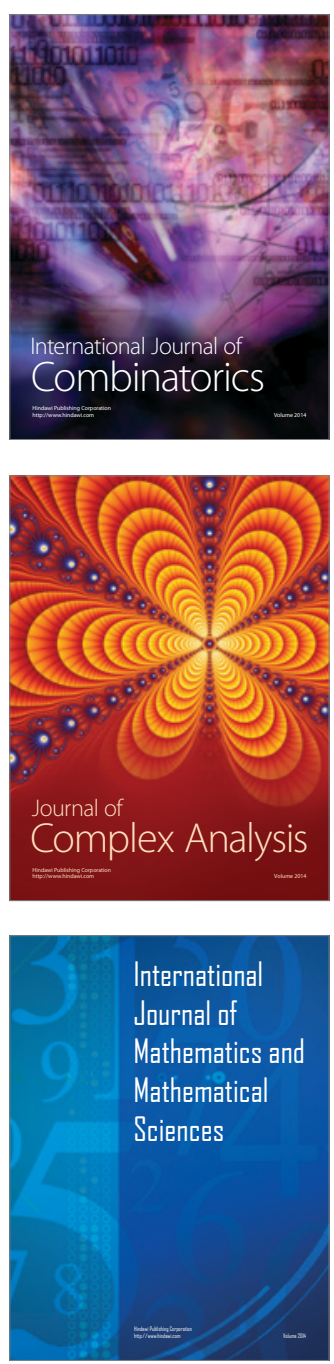
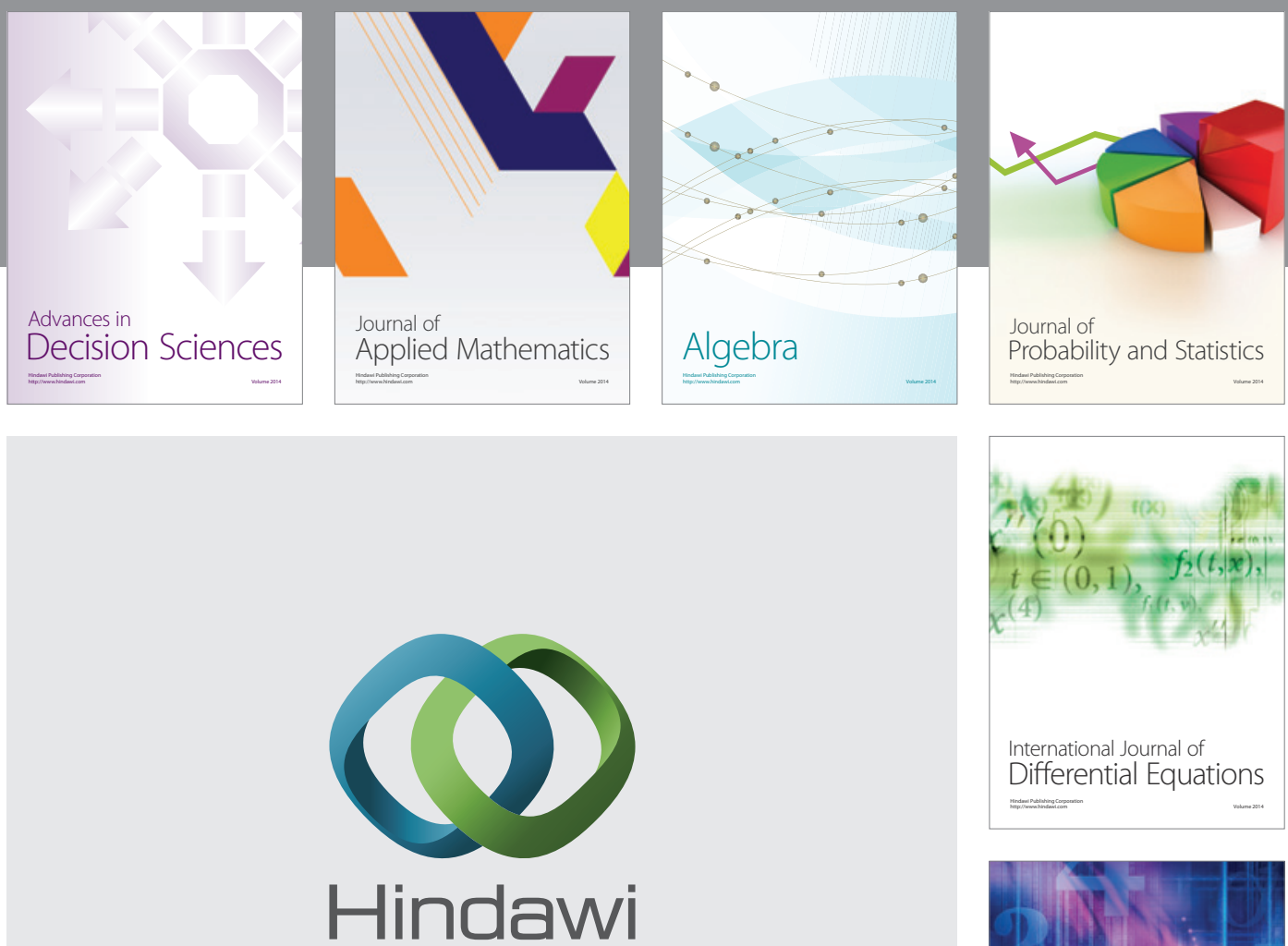

Submit your manuscripts at http://www.hindawi.com
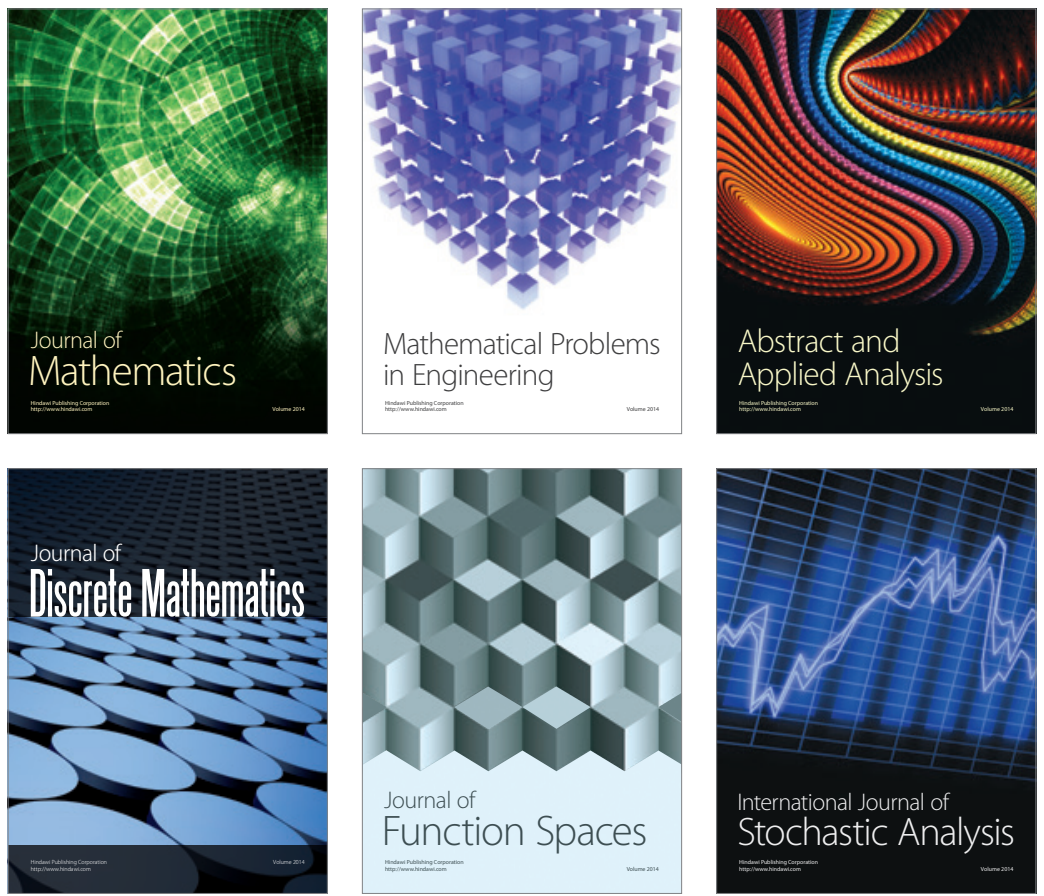

Journal of

Function Spaces

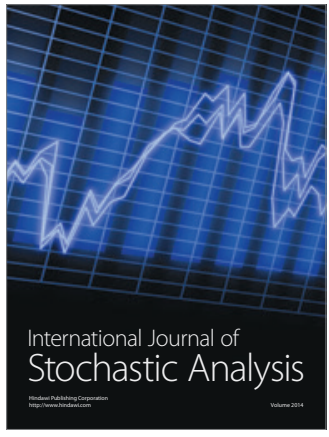

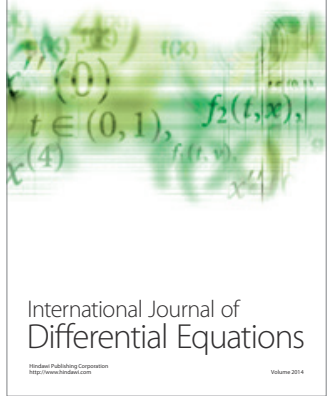
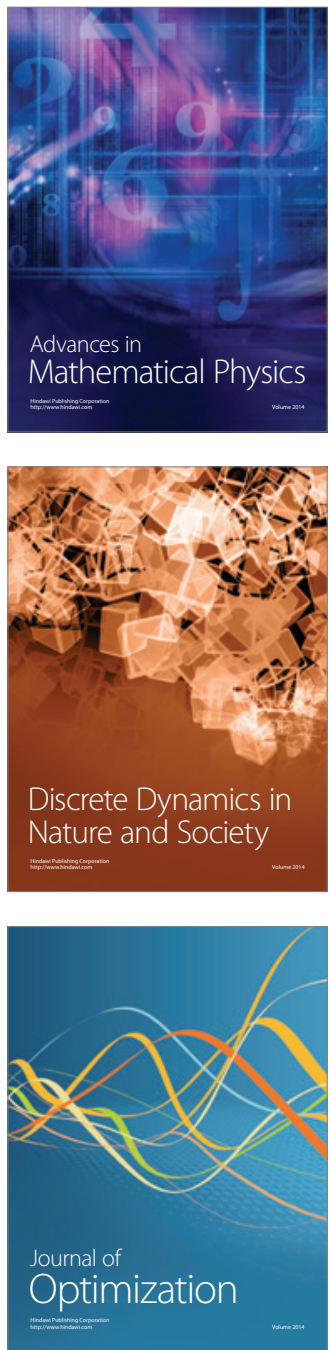